\title{
Radiosensitivity of Human Breast Cancer Cell Lines Expressing the Breast Tumor Kinase (Brk)
}

\author{
Bourton EC 1 , Hussain $\mathrm{H}^{1}$, Plowman $\mathrm{PN}^{2}$, Harvey $\mathrm{AJ}^{1 *}$ and Parris $\mathrm{CN}^{1 *}$
}

${ }^{1}$ Division of Biosciences, Department of Life Sciences, College of Health and Life Sciences, Brunel University London, Kingston Lane, Uxbridge, Middlesex UB8 3PH, UK ${ }^{2}$ Department of Radiotherapy, St Bartholomew's Hospital, West Smithfield, London, EC1A 7BE, UK

\begin{abstract}
The breast tumor kinase (Brk) is over-expressed in $80 \%$ of all breast cancers and we sought to determine the influence of this oncogene on radiation sensitivity in breast cancer cell lines. Since radiotherapy is a routinely used method of treatment for early and intermediate stage breast cancer, the alteration of clinical radiosensitivity in breast cancer by an oncogene over-expression would have important implications in radiotherapy management. To address this question, we conducted an in vitro study of radiation sensitivity in two breast cancer cell lines, MDA-MB-157 and MDA-MB-468 transfected with cDNA constructs to over-express the following genes: Brk wild type (WT); Brk with an inactivating mutation in the kinase domain (KM) and vector only. Gamma H2AX foci assays by imaging flow cytometry were used to measure DNA double strand break (DSB) repair after radiation exposure. Total ataxia telangiectasia (ATM) and activated phospho794-ATM protein was measured by imaging flow cytometry. In all cell lines tested there was a proportionate decline in cell survival following gamma radiation exposure. Radiation sensitivity of the cell lines in clonogenic assays and repair of DNA double strand breaks were similar and independent of Brk expression status. We conclude that over-expression of the Brk proto-oncogene in breast cancer cell lines does not appear to influence radiation sensitivity or affect DNA DSB repair.
\end{abstract}

Keywords: Breast tumor kinase; $\gamma-\mathrm{H} 2 \mathrm{~A} \Xi$; Radiosensitivity; DNA repair; Ataxia telangiectasia (ATM)

Abbreviations: ATM gene: Ataxia Telangiectasia Mutated gene; ATCC: American Tissue Culture Collection; Brk: Breast Tumor Kinase; CDK; Cyclin Dependent Kinase; DSB: Double Strand Break; DMEM: Dulbecco's Modified Eagle Medium; DNA: Deoxyribose Nucleic Acid; Gy: Gray (100 Rads Irradiation); Mtor: Mammalian Target of Rapamycin; NHEJ: Non Homologous End Joining DNA Repair; RPMI; Roswell Park Memorial Institute Cell Growth Medium; SF2: Surviving Fraction of Cells After Exposure to 2 Gy Gamma Radiation

\section{Introduction}

Collectively, breast cancers are the most common forms of cancer in women with over 1.676 million new diagnoses world-wide each year [1]. Since the introduction of national screening programmes, effective clinical staging, molecular diagnosis together with efficient treatment regimens, the survival of patients with breast cancer has improved significantly In the United Kingdom [1].

Radiotherapy is an important component of multimodal therapy for women with early stage (I and II) breast cancer [2]. In the UK all women should be offered radiotherapy following surgery or chemotherapy $[3,4]$ and National Institute for Health Care Excellence (NICE) guidelines recommend fractionated radiotherapy of 40 Gy radiation in 15 fractions [5].

The intracellular protein kinase Brk (also known as breast tumor kinase or protein tyrosine kinase 6, PTK6) has been implicated in the development and progression of a number of different tumor types including breast cancer and is over-expressed in $80 \%$ of all types of breast cancer [6]. In this study we wished to determine the relationship between Brk oncogene over-expression and radiation sensitivity in breast cancer cell lines. Brk has been shown to be up-regulated and overexpressed in up to $80 \%$ of ductal breast carcinomas but until recently was not thought to be expressed in normal breast tissue [7-9]. Brk is a non-membrane bound tyrosine kinase and has been demonstrated to interact with a number of key intracellular cell signalling proteins which lead to increased cell survival, enhanced cellular migration and elevated proliferation [7]; however, the impact of Brk expression on cellular radiation sensitivity and DNA DSB repair has not been evaluated. Previous studies do not reveal any direct link of Brk with DNA repair processes in breast cancer, although Brk expression has been linked to regulation of apoptosis in response to DNA damaging agents in non-tumor cells [9-11]. Also elevated Brk expression leads to increased ErbB signalling via PI3-K/AKT [12], an upstream activator of the mTOR (mammalian target of rapamycin) pathway [13]. PI3-K/ $\mathrm{AKT} / \mathrm{mTOR}$ is considered as a potential therapeutic target in breast cancer [13]. Moreover, elevated mTOR signalling is known to suppress the ataxia telangiectasia mutated protein (ATM) expression by inducing microRNAs targeting ATM for destruction [14]. ATM is a key cell cycle regulator and mutational inactivation leads to extreme cellular and clinical sensitivity to radiation [15]. Thus we may hypothesise that increased Brk expression in breast cancer cells may affect the response of breast cancer cells to radiation via the ATM pathway. To examine the relationship between cellular response to ionising radiation and $\mathrm{Brk}$ expression in breast cancer cells we analysed the response of two groups of breast cancer cell lines. The triple negative breast cancer cell lines

*Corresponding authors: Christopher N. Parris, Division of Biosciences, Department of Life Sciences, College of Health and Life Sciences, Brunel University London, Kingston Lane, Uxbridge, Middlesex UB8 3PH, UK, Tel: +44 (0)1895 266293; Fax: +44 (0)1895 269873; E-mail: christopher.parris@brunel.ac.uk

Amanda J. Harvey, Division of Biosciences, Department of Life Sciences, College of Health and Life Sciences, Brunel University London, Kingston Lane, Uxbridge Middlesex UB8 3PH, UK, Tel: +44 (0)1895 267264; Fax: +44 (0)1895 269873; E-mail: amanda.harvey@brunel.ac.uk

Received January 23, 2015; Accepted March 03, 2015; Published March 05 2015

Citation: Bourton EC, Hussain H, Plowman PN, Harvey AJ, Parris CN (2015) Radiosensitivity of Human Breast Cancer Cell Lines Expressing the Breast Tumor Kinase (Brk). J Cancer Sci Ther 7: 095-101. doi:10.4172/1948-5956.1000331

Copyright: (c) 2015 Bourton EC, et al. This is an open-access article distributed under the terms of the Creative Commons Attribution License, which permits unrestricted use, distribution, and reproduction in any medium, provided the original author and source are credited. 
MDA-MB-157 and MDA-MB-468 are derived from basal-type breast cancers and express low to undetectable levels of Brk [16,17]. These were transfected with plasmid vectors to over-express either the wild type Brk cDNA, a cDNA expressing a Brk protein with an inactivating mutation in the kinase domain of the protein (Brk-KM), or the empty plasmid vector. In the MDA-MB-157 cell line we observed significantly elevated cellular radiation resistance in the cells over-expressing the wild type Brk protein when compared to the cells transfected with the inactivated Brk-KM counterpart cDNA or the cells transfected with the empty plasmid vector. However, in the MDA-MB-468 cell lines, increased Brk expression was not associated with increased cellular resistance to radiation.

\section{Materials and Methods}

\section{Transfected cell lines}

Three variants each of the cell lines MDA-MB-157 and MDAMB-468 were used, namely -BRK-WT (containing the wild-type Brk cDNA), -BRK-KM (containing a mutated Brk cDNA), and -Vector (containing the empty vector $\mathrm{pRc} / \mathrm{CMV}$ ). The cell lines were originally obtained from ATCC, the transfections were carried out using Fugene-6 (Roche, Welwyn Garden City, UK) as part of our previous studies, and the stable cell lines were maintained in their usual culture medium supplemented with $400 \mu \gamma / \mu \lambda$ G418 (Melford Laboratories Ltd, Ipswich, UK) $[9,18]$.

\section{Cell culture}

The cell lines were routinely cultured in T75 cell culture flasks (Sarstedt Ltd, Leicester, UK) in Dulbecco's modified Eagle's medium (MDA-MB-157 and MDA-MB-468) or RPMI-1640 (Lab Tech Ltd, East Sussex, UK) (T-47D, BT474, Sk-Br-3 for western blot analysis) supplemented with $10 \%$ foetal bovine serum, $2.0 \mathrm{mM} \mathrm{L}$-Glutamine (Labtech) and $100 \mathrm{Uml}^{-1}$ Penicillin and Streptomycin (Scientific Laboratory Supplies Ltd., Leicester, UK). GI101 cells were cultured in RPMI-1640 supplemented as described plus $5 \mu \mathrm{g} / \mathrm{ml}$ insulin (Sigma-Aldrich Ltd., Dorset, UK). MCF10A were routinely cultured in 1:1 HamsF12: DMEM (Life Technologies Ltd., Paisley, Scotland) supplemented with $10 \%$ foetal bovine serum, $2.0 \mathrm{mM} \mathrm{L}$-Glutamine and $100 \mathrm{Uml}^{-1}$ penicillin and streptomycin plus $1 \mu \mathrm{g} / \mathrm{ml}$ hydrocortisone, 5 $\mathrm{ng} / \mathrm{ml} \mathrm{EGF}$ and $5 \mu \mathrm{g} / \mathrm{ml}$ insulin (Sigma-Aldrich).

Cells were incubated at $37^{\circ} \mathrm{C}$ in a humidified atmosphere of $5 \%$ $\mathrm{CO} 2$ in air. At approximately $80 \%$ confluence cells were routinely subcultured by trypsinisation in trypsin/EDTA solution containing 170 $\mathrm{U} / \mathrm{ml}$ trypsin, $0.2 \mathrm{mg} / \mathrm{ml}$ EDTA in phosphate buffered saline (Labtech).

Cell counts and viability were determined using a "Countess" automated cell counter that utilises the trypan blue exclusion method (Life Technologies). Cells were not used for experimentation if overall viability was less than $80 \%$. To minimise genetic drift in culture all cells were used over a restricted range of ten passages.

\section{Clonogenic cell survival assays}

Clonogenic assays post-exposure to gamma radiation were conducted as described previously [19]. Briefly, cells were suspended in appropriate complete medium and an aliquot retained as an unirradiated control. The remaining aliquots were irradiated with 2, 4, 6 and 8 Gy gamma radiation from a 60Cobalt source (Puridec Technologies Ltd., Oxon, UK) sited at a distance of $25 \mathrm{~cm}$ from the source with a dose rate of $0.8 \mathrm{~Gy}$ per minute. Cells from the samples were plated into each of three $10 \mathrm{~cm}$ dishes, containing $10 \mathrm{ml}$ DMEM complete medium. The cell suspension concentrations were: Control and 2 Gy (1000 cells/plate); 4 Gy (2000 cells/plate); 6 Gy (3000 cells/ plate); $8 \mathrm{~Gy}$ (4000 cells/plate). Cells were incubated for three weeks in a humidified atmosphere at $37^{\circ} \mathrm{C}$ with $5 \% \mathrm{CO}_{2}$ in air, fixed in industrial methylated spirits, stained with $1.0 \%$ methylene blue in water (SigmaAldrich). The number of colonies of greater than 50 cells was counted. The number of colonies derived from irradiated cells was expressed as a percentage of colonies in the un- irradiated control dishes.

\section{DNA damage induction by gamma radiation exposure}

Cells were plated into $10 \mathrm{~cm}$ dishes at concentrations of $2 \times 10^{6}$ cells/dish (for the determination of DNA DSB by $\gamma-\mathrm{H} 2 \mathrm{~A} \Xi$ induction) and $4 \times 10^{6}$ cells/dish (for the identification of total or phosphorylated ataxia telangiectasia protein levels), and left overnight in an incubator (conditions described previously).

One dish of each cell line was retained as an un-irradiated control; the remaining dishes were irradiated with $2 \mathrm{~Gy}$ gamma radiation from a 60Cobalt source (Puridec) sited at a distance of $25 \mathrm{~cm}$ with a dose rate of 0.8 Gy per minute. Two Gray gamma irradiation was chosen to correspond to fractionated doses of radiation used in clinical protocols. We have also demonstrated previously that the induction of DNA DSB by 2 Gy gamma irradiation represents a region of maximal $\gamma-\mathrm{H} 2 \mathrm{~A} \Xi$ induction [20]. Following exposure the cells were returned to the incubator (incubation conditions described previously), and one dish removed at 30 minutes, 3 hours, 5 hours and 24 hours after irradiation.

Cells in each dish were harvested, washed once with ice-cold PBS (Severn Biotech Ltd., Worcestershire, UK) and fixed in ice-cold methanol:acetone $(50: 50 \mathrm{v} / \mathrm{v})$. Two compensation samples for imaging flow cytometry were prepared in the same way at the 30 minute timepoint. Samples were stored at $-20^{\circ} \mathrm{C}$ until the immunocytochemistry stage. The immunocytochemistry commenced within 5 days of irradiation.

\section{Immunocytochemistry - gamma $\mathrm{H} 2 \mathrm{AX}$ assay}

Cells were re-suspended in ice-cold PBS then agitated gently for 5 minutes at room temperature in permeabilisation buffer consisting of $0.5 \%$ Triton $^{\text {Tw }} \mathrm{X}-100$ (Sigma-Aldrich Ltd) in PBS. Cells were incubated with gentle agitation at room temperature for 1 hour in blocking buffer consisting of $5.0 \%$ rabbit serum (Labtech) with $0.1 \%$ Triton $^{\text {m }}$ X-100 in PBS. After the removal of the blocking buffer the cells were incubated for $24 \mathrm{hrs}$ with gentle agitation at $4^{\circ} \mathrm{C}$ in primary antibody solution, consisting of an anti-phospho-histone H2AX (serine139) mouse monoclonal IgG1 antibody, clone JBW301 (Millipore Ltd., Watford, UK) diluted 1 in 10,000 in blocking buffer. Excess primary antibody was removed by washing twice in buffer consisting of $0.1 \%$ Triton $^{\text {Tx }}$ X-100 in PBS. Primary antibody labelled cells were incubated in secondary antibody solution consisting of Alexa Fluor 488 rabbit anti-mouse IgG antibody (Invitrogen) diluted 1 in 1000 in blocking buffer. This was added to each sample, except the DRAQ5 $5^{\text {min }}$ (Biostatus Ltd., Leicester, UK) compensation samples, and incubated with gentle agitation for 1 hour at room temperature. Excess secondary antibody was removed by washing twice with wash buffer. The cells were resuspended in $100 \mu$ l. Accumax ${ }^{\text {tix }}$ solution (Labtech) and left overnight at $4^{\circ} \mathrm{C}$ (no agitation). Five $\mu \mathrm{M}$ DRAQ $5^{\mathrm{Tx}}$ solution was added to each sample, except the AlexaFluor 488 compensation samples. The images of at least 10,000 cells for each time point were collected by imaging flow cytometry.

\section{Determination of total and phosphorylated ataxia telangiectasia (ATM) mutated protein in the cell lines}

To determine the relationship between Brk expression and total 
and phosphorylated ATM protein in the breast cancer cell lines, cells were irradiated with 2 Gy gamma radiations (as described earlier). In un-irradiated cells and at 30 minutes after radiation exposure cells were fixed in 50:50 (v/v) ice-cold methanol:acetone. After rehydration of the cells and blocking in block buffer (as described for $\gamma-\mathrm{H} 2 \mathrm{~A} \Xi$ staining), cells were labelled with a primary antibody, either a mouse monoclonal antibody for the C-terminal region of the ATM protein (to detect total ATM), or a rabbit polyclonal for phosphorylated serine794 of the ATM protein (to detect phosphorylated ATM). Both primary antibodies (ECM Biosciences, 2B Scientific Ltd, Upper Heyford, Oxon, UK) were diluted 1 in 500 in blocking buffers, and the cells were incubated in the presence of the primary ATM antibody for $24 \mathrm{hrs}$ at $4^{\circ} \mathrm{C}$ with gentle agitation. Following two 5 minute washes with wash buffer, cells were stained with either a rabbit anti-mouse or a goat anti-rabbit secondary antibody solution consisting of Alexa Fluor 488 IgG antibody diluted 1 in 1000 in blocking buffer. This was added to each sample, except the DRAQ5 ${ }^{\mathrm{Tm}}$ compensation samples, and incubated with gentle agitation for 1 hour at room temperature. Cells were washed twice in wash buffer and left overnight at $4^{\circ} \mathrm{C}$ in Accumax $^{\mathrm{mm}}$. Five micromole DRAQ5 ${ }^{\mathrm{m} w}$ solution was added to each sample, except the AlexaFluor 488 compensation samples.

\section{Imaging flow cytometry}

Imaging flow cytometry was carried out using the ImagestreamX system (Amnis Inc., Seattle, Washington, USA) as described previously $[20,21]$. Images of 10,000 cells were captured on channel 1 for brightfield (BF); on channel 2 for Alexa Fluor 488 (AF), depicting staining of $\gamma-\mathrm{H} 2 \mathrm{AX}$ foci or ATM protein localisation; on channel 5 for DRAQ5 ${ }^{\mathrm{Tw}}$ (D5), depicting staining of the nucleus. Cell classifiers were applied to the BF channel to capture objects that represented only single cells, where an empirically determined scale of 50 as the lower limit and 300 as the higher limit was used. Cellular fluorescence $(\gamma-\mathrm{H} 2 \mathrm{AX}$ foci or ATM protein localisation) was detected by excitation with a $488 \mathrm{~nm}$ laser at a minimum power setting of $70 \mathrm{~mW}$; all images were captured using a $40 \times$ objective lens. Images of cells were acquired at a rate of 100 -200 cell images per second.

\section{Image compensation}

Image compensation has been described in detail previously elsewhere $[20,21]$. In brief, cells that had been fixed 30 minutes postirradiation where $\gamma-\mathrm{H} 2 \mathrm{~A} \Xi$ staining or ATM protein staining intensity was likely to be highest were used to capture images of cells with the $488 \mathrm{~nm}$ laser only and without brightfield illumination. Cells that were stained with antibody (Alexa Fluor488) only or DRAQ5 ${ }^{\mathrm{mm}}$ only were used for generating the compensation matrix. The Ideas ${ }^{\mathrm{Tx}}$ software compensation wizard produces a table of coefficients whereby detected light produced by each cellular image is placed into the proper channel (channel 2 for antibody staining and channel 5 for DRAQ $5^{\text {rux }}$ ) on a pixel-by-pixel basis. The coefficients were normalized to 1 and each coefficient represents the leakage of fluorescent signal into juxtaposed channels. Calculated compensation values were applied to all subsequent analyses as appropriate.

\section{Analysis of cell images - calculation of gamma H2AX foci number in cells}

Gamma-H2AX foci were quantified in approximately 10,000 images of cells captured with the Inspire ${ }^{\mathrm{m} w}$ imaging flow cytometry software using a method described in detail elsewhere [8,12]. In brief, the spot counting wizard integral to the Ideas ${ }^{\mathrm{Tn}}$ image analysis software was employed. Here a population of cells with very few foci $(<3)$ and very high foci numbers $(>10)$ are user identified and then employed by the software to calculate the number of foci in each cellular image. From this analysis the average number of foci in all 10,000 cells can be determined.

\section{Analysis of cell images - ATM protein staining in cells}

To quantitate either the cellular level of total ATM or the phosphoserine784 ATM levels in irradiated and non-irradiated cells, the nuclear region of the cells was identified using the masking feature of the Ideas $^{\mathrm{Tm}}$ software programme. The intensity of Alexa Fluor488 staining (total ATM or phosphoserine784 ATM) was calculated by determining the intensity of channel 2 staining within the nucleus. The mean levels of antibody staining here represent the ATM protein levels in these cells.

\section{Statistical analysis}

Statistical analysis was carried out using the data analysis feature in Microsoft Excel. Two-way analysis of variance was used to compare clonogenic cell survival following exposure to the sub-lethal dose of 2 Gy gamma irradiation. Similarly, distribution of foci in cell lines post radiation exposure in the presence or absence of Brk expression was compared using the Student's unpaired t-test, a p-value of $<0.05$ being regarded as significant.

\section{Western blotting}

Cells were cultured until monolayers were $80 \%$ confluent, washed in PBS and harvested as described above. Following counting and centrifugation, cell pellets were lysed in $100 \mu \mathrm{l}$ hot SDS-PAGE lysis buffer per 106 cells, boiled for 5 minutes and stored at $-20^{\circ} \mathrm{C}$. Protein lysates equivalent to $1.5 \times 10^{5}$ cells were separated on a $10 \%$ SDSpolyacrylamide gel and electro-blotted onto nitrocellulose membrane in $1 \mathrm{x}$ Towbin transfer buffer.

Membranes were blocked in 5\% non-fat skimmed milk/TBS-T for 1 hour and then incubated overnight at $4^{\circ} \mathrm{C}$ in primary antibody in the same blocking buffer (anti-GAPDH - Abcam Ltd., Cambridge, UK) or in 5\% BSA in TBS-T (anti-Brk - ICR100) [22]. Following washes in TBS-T, the proteins were visualised on film using an appropriate hrp-conjugated secondary antibody (Dako Ltd., Cambridge, UK) with chemiluminescent substrate.

\section{Results}

\section{Clonogenic cell survival gamma radiation - MDA-MB-157 cells}

Figure 1A shows the survival of the cell lines following exposure to increasing doses of gamma radiation from a 60Cobalt source. Figure 1A shows the survival of the MDA-MB- 157 cell lines expressing either Brk-WT, Brk-KM or an empty plasmid vector. For all cell lines there was a proportionate decline in cell survival following exposure to increasing doses of gamma radiation. In the Brk-WT cell line the cells were more resistant to the lethal effects of gamma radiation compared to Brk-KM and Vector cell lines. By comparing the SF2 values (the survival of the cells following exposure to 2 Gy gamma radiation), the Brk-WT cell lines displayed an SF2 of $75.35 \%+/-7.62$ standard error (SE). In the Brk-KM cell line the SF2 value was 50.56\% +/- $0.39 \mathrm{SE}$ and for the vector cell line the SF2 is $30.81+/-10.84$ SE. Therefore the BrkWT cell line was 1.40 and 2.28 times more resistant to the lethal effects of gamma radiation than the Brk-KM and vector cell line variants respectively. By comparing the individual SF2 values from at least three replicate cell survival experiments, using a Student's unpaired T-test, 


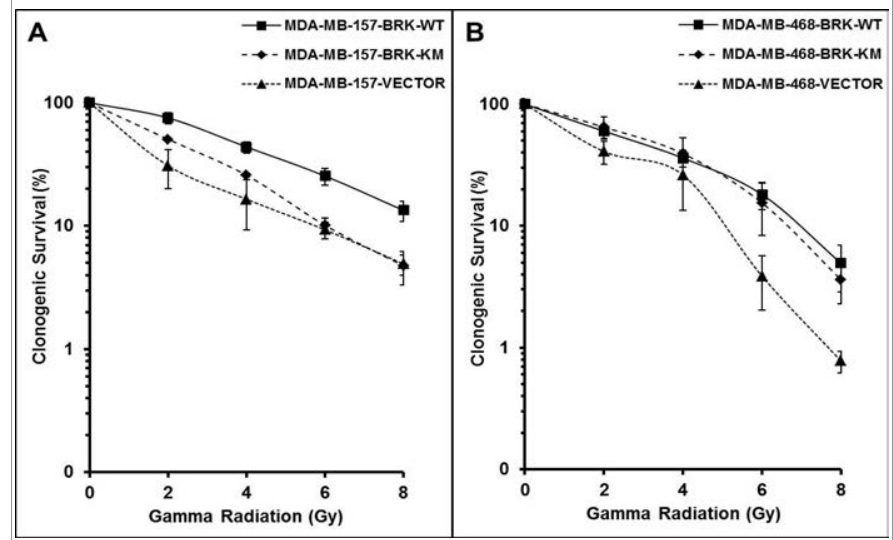

Figure $1 \mathrm{~A}$ and $\mathrm{B}$ : Show the clonogenic cellular sensitivity of the two breast cancer cell lines sets (Brk-WT, Brk-KM, Vector) to increasing doses of gamma radiation. Figure $1 \mathrm{~A}$ shows the response of the MDA-MB-157 cell lines and Figure 1B shows the response of the MDA-MB- 468 cell lines. The data are derived from at least three independent experiments for each cell line. Error bars represent standard error of the mean cellular survival after exposure to 2, 4, 6 and 8 Gy gamma radiations.

the Brk-WT cell line was significantly more resistant to the gamma radiation than the Brk-KM and Vector cell lines (Student's Unpaired t-test $\mathrm{p}>0.05)$.

\section{Clonogenic cell survival gamma radiation - MDA-MB-468} cells

In Figure 1B the survival of three MDA-MB-468 cell lines is shown. Similar to the MDA-MB-157 cell lines the MDA-MB-468 cell lines display a proportionate reduction in clonogenic cell survival following exposure to increasing doses of gamma radiation. Unlike the MDAMB-157 cell lines the MDA-MB-468 cells expressing Brk-WT or Brk$\mathrm{KM}$ have identical responses to the lethal effects of gamma radiation. Here the SF2 values for both cells lines were $60.03 \%+/-8.03$ SE for Brk-WT and $64.79 \%+/-13.45$ SE for the Brk-KM cell line. The MDAMB-468 vector cells exhibited an SF2 value of $40.84 \%+/-8.81$ SE. Therefore the Brk-WT and Brk-KM cells were 1.4 fold more resistant to the lethal effects of gamma radiation than the Vector cells. A Student's unpaired t-Test used to compare the SF2 values for the MDA-MB-468 cell lines shows that there is no significant difference in the survival of the Brk-WT and Brk-KM and vector only cell lines ( $p=0.385)$.

\section{Gamma-H2AX foci induction in the breast cancer cell lines}

In Figures $2 \mathrm{~A}$ and $\mathrm{B}$ the induction of $\gamma-\mathrm{H} 2 \mathrm{~A} \Xi$ foci in the MDAMB-157 (Figure 2A) and MDA-MB-468 cells lines (Figure 2B) is shown. Common to both cell lines irrespective of Brk expression status was the typical and rapid induction of DNA DSB revealed by a concomitant elevation of foci in the nuclei of cells. Then, over a $24 \mathrm{hrs}$ period there was a reduction of foci to un-irradiated levels. In the MDA-MB-157 cell line group there was a similar pattern of foci induction. We had originally hypothesised that the radiation resistant Brk-WT expressing cell line would reveal an altered DSB repair profile compared to the Brk-KM and Vector cell line variants. However, in three replicate experiments, the numbers of foci were not significantly different unirradiated cells for all three variants $(\mathrm{p}<0.05)$. Similarly at 30 minutes post irradiation, the point at which foci induction is maximal, there were similar levels of foci induction ranging between $5.9+/-0.78 \mathrm{SE}$ and 7.84 foci $+/-1.90$ SE which were not significantly different $(\mathrm{p}<0.05)$. Moreover, over the course of $24 \mathrm{hrs}$ post irradiation the kinetics of
DSB repair in the three cell line variants was not significantly different $(\mathrm{p}<0.05)$, and at $24 \mathrm{hrs}$ after irradiation the number of foci in each of the three cell lines ranged from 3.46+/- 0.52 SE for the Brk-WT and $3.90+/-0.32 \mathrm{SE}$ for the Vector cell line.

In the MDA-MB-468 group of cell lines a similar picture of DSB repair is shown. Typically at 30 minutes post-irradiation, there was a maximal induction of DSB revealed by high levels of foci number and this returned to normal un-irradiated levels at $24 \mathrm{hrs}$ after irradiation. Again and in a similar manner to the MDA-MB-157 cell line group, there were no significant differences in the kinetics of repair of DSB over a $24 \mathrm{hrs}$ period within the three cells lines irrespective of Brk functional status $(\mathrm{p}<0.05)$.

Total ATM and ATM phosphoserine794 in cells before and after exposure to $2 \mathrm{~Gy}$ gamma radiation

To determine if there was a functional relationship between total and phosphoserine794 ATM and Brk expression, the levels of both ATM protein variants were determined by immunological staining of total and phosphorylated ATM followed by analysis by imaging flow cytometry. For both total and ATM-phosphoserine794, the level of fluorescence in un-treated cells was compared to the levels in cells exposed to 2 Gy gamma radiation. We hypothesised that in cells

A

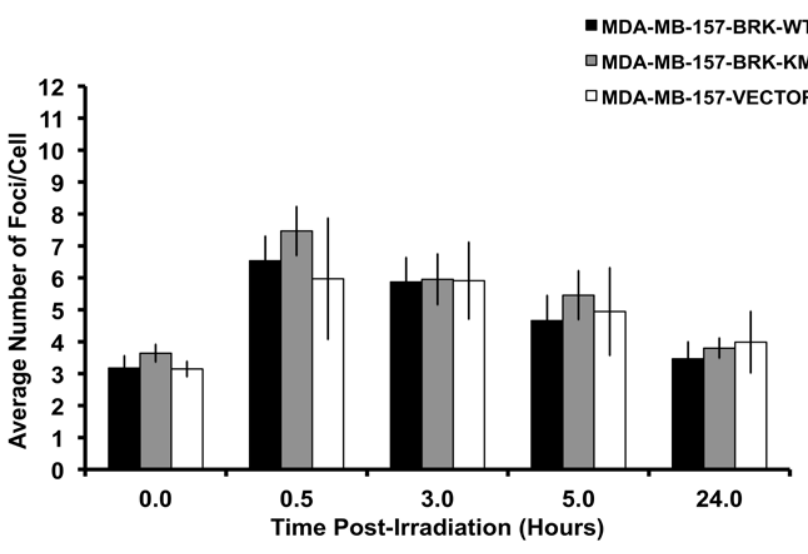

B

MDA-MB-468-BRK-WT 口MDA-MB-468-BRK-KM

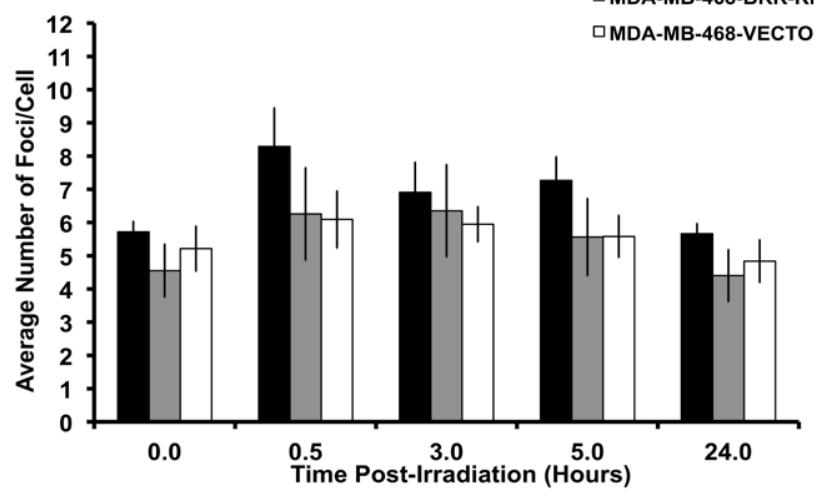

Figure 2A and B: Show the kinetics of DNA double strand break repair after exposure of the cells to $2 \mathrm{~Gy}$ gamma radiation. DSB were measure by nuclear $\mathrm{Y}-\mathrm{H} 2 \mathrm{AX}$ foci induction which is representative of DSB. $\mathrm{Y}-\mathrm{H} 2 \mathrm{AX}$ foci were measured in untreated cells and those exposed to 2 Gy gamma radiations at $0.5,3,5$ and 24 hours post treatment. Figure $2 \mathrm{~A}$ shows the data for the MDA-MB-157 cell lines and Figure 2B for the MDA-MB-468 cell lines. Data are derived from at least three independent experiments. Error bars show standard error of the mean foci induction at each time point. 
Citation: Bourton EC, Hussain H, Plowman PN, Harvey AJ, Parris CN (2015) Radiosensitivity of Human Breast Cancer Cell Lines Expressing the Breast Tumor Kinase (Brk). J Cancer Sci Ther 7: 095-101. doi:10.4172/1948-5956.1000331

expressing the Brk-WT protein there would be altered levels of ATM and more importantly ATM-phosphoserine794 due to higher levels of functional Brk-WT protein.

The levels of total ATM for both the MDA-MB-157 and MDAMB-468 cells are shown in Figures $3 \mathrm{~A}$ and $\mathrm{B}$ respectively. For the MDAMB-157 cell lines (Figure 3A) the level of fluorescence representing total ATM in un-irradiated cells is shown as a relative fluorescence of 1.0. The amount of total ATM following 2 Gy gamma radiations was presented in comparison to the un-irradiated cells. In the Brk-WT cell lines the level of ATM slightly increased from 1.0 to 1.08 relative fluorescence. However in the Brk-KM and Vector cell lines there was a reduction of total ATM when compared to Brk-WT cells.

For the MDA-MB-468 cell lines (Figure 3B), there was a reduction in total cellular ATM following 2 Gy gamma radiation exposures in the Brk-WT and Brk-KM cells but levels were unchanged in the Vector cells.

The levels of phosphorylated ATM serine794 for both the MDAMB-157 and MDA-MB- 468 cells are shown in Figures $4 \mathrm{~A}$ and $\mathrm{B}$ respectively. Interestingly, there seemed to be no relationship between phosphorylated ATM serine794 and overall cellular radiation sensitivity. Following 2 Gy exposures the levels declined in the MDA-

\section{A}

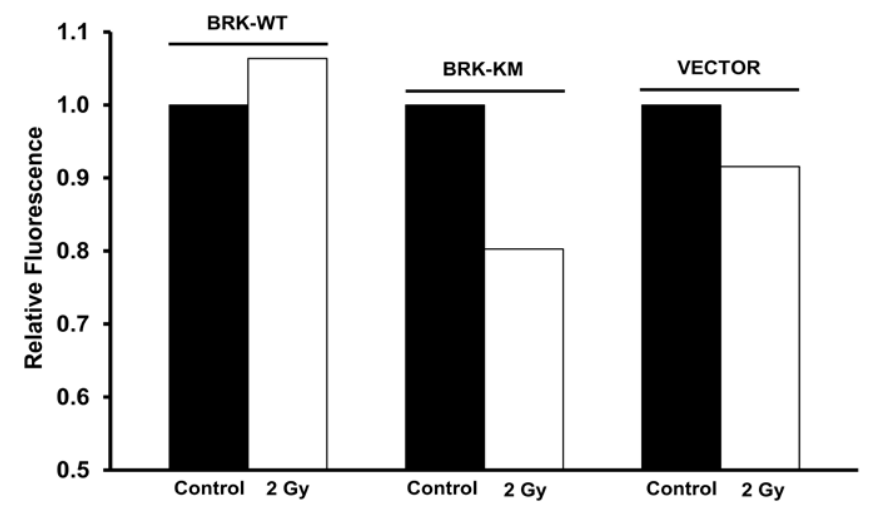

B

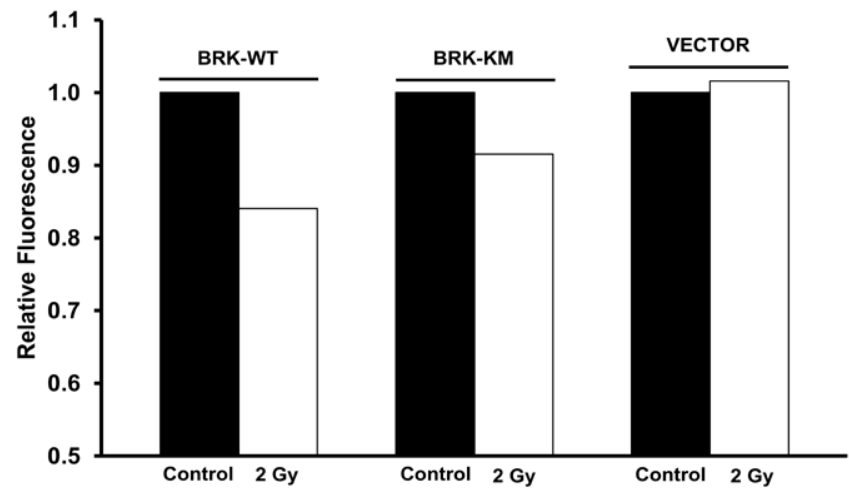

Figure $3 \mathbf{A}$ and B: Show the total level of ATM protein in the cell lines in untreated cells and in those directly following their exposure to $2 \mathrm{~Gy}$ gamma radiations. Data are derived from analysing the nuclear intensity of fluorescent immunostaining of the ATM protein derived from imaging flow cytometry of at least 10,000 cells. Figure $3 A$ shows the ATM protein in the MDA-MB-157 cell lines and Figure 3B for the MDA-MB-468 cell lines.
A

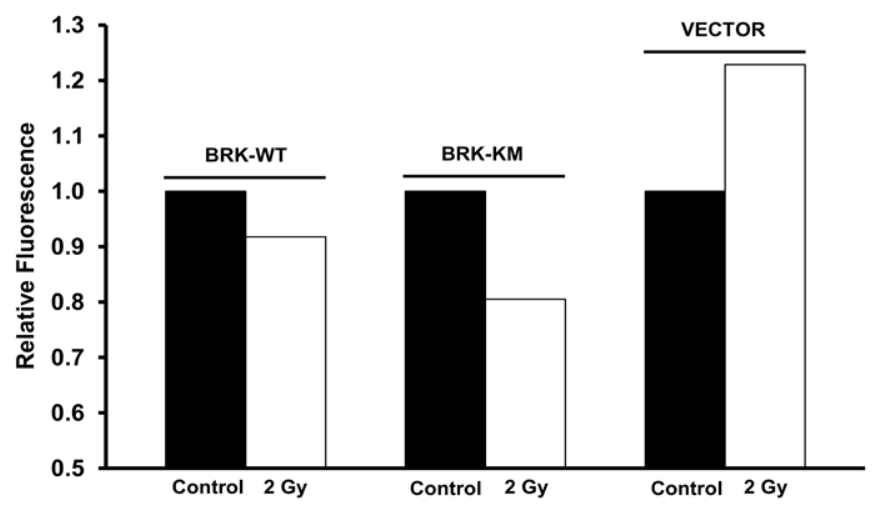

B

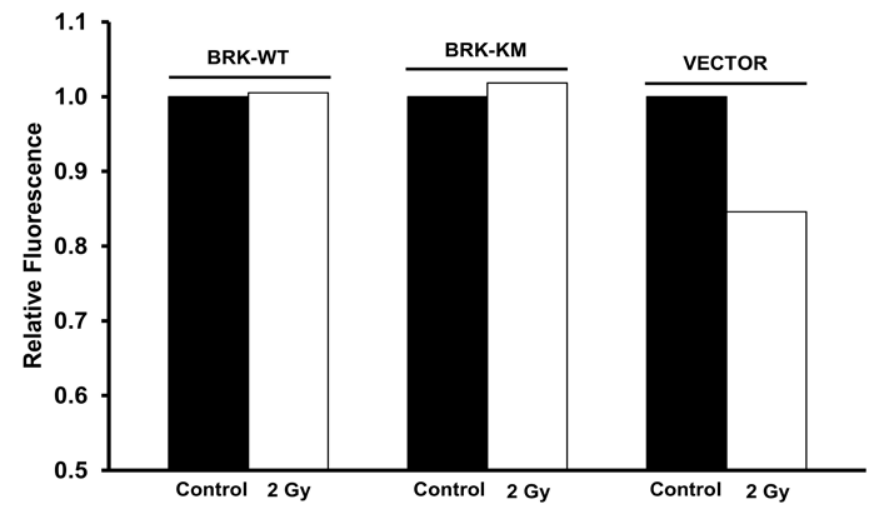

Figure 4A and B: Show the level of ATM phosphoserine794 protein in the cell lines before and after exposure to 2 Gy gamma radiations. Data are derived from analysing the nuclear intensity of fluorescent immunostaining of ATM phosphoserine794 protein derived from imaging flow cytometry of at least 10,000 cells. Figure 4A shows the ATM phosphoserine794 protein in the MDA-MB-157 cell lines and Figure 4B in the MDA-MB-468 cell lines.

MB-157 Brk-WT and Brk-KM cell lines but increased in the Vector cell line (Figure 3A).

For phosphorylated ATM794 in the MDA-MB-468 cells, there were unchanged levels in the Brk-WT and Brk-KM cells after 2 Gy exposures when compared to untreated control cells. However in the Vector cell line there was a nearly $50 \%$ reduction in phosphorylated ATM794. The functional relevance of this data will be discussed.

Given that expression of WT-Brk decreased MDA-MB-157 cell sensitivity to radiation, we sought to determine whether Brk plays a role in radiation sensitive in a panel of breast cancer cells lines derived from different tumor types (Figure 5A and B). Clonogenic assays were carried out on all the cell lines, and their SF2 (the survival of the cells following exposure to 2 Gy gamma radiation) was calculated (Figure 6 upper panel). Brk was detected in whole cell lysates from these cell lines by western blotting; however, there was no correlation between sensitivityand Brk expressions was observed (Figure 6 lower panel).

\section{Discussion}

In this report we have examined the influence of the Brk kinase on the radiosensitivity of cells lines derived from human breast cancer. We have also investigated the biochemical relationship between Brk 


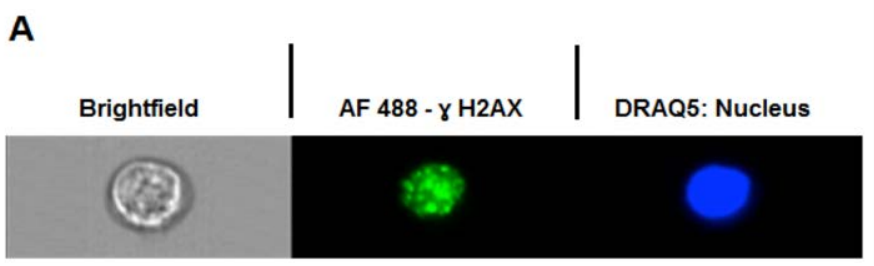

B

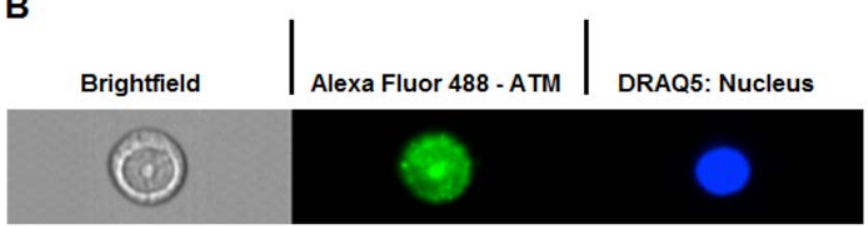

Figure 5A and B: Show representative images of imaging flow cytometry of $\mathrm{Y}-\mathrm{H} 2 \mathrm{AX}$ foci staining (A) and total ATM staining (B). Three channels are presented whereby channel one represents the brightfield image; channel two the immune-labelled $y-H 2 A X$ foci or ATM protein and channel 5 shows the DRAQ5 staining of the nucleus within the cells.

SF2
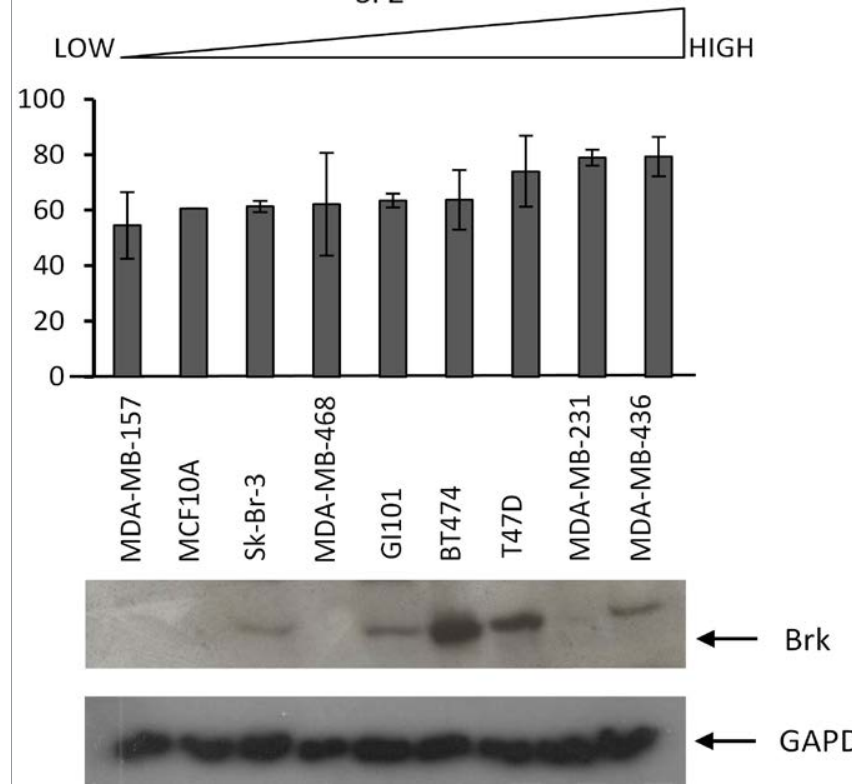

GAPDH

Figure 6: Shows the levels of Brk expression related to SF2. Clonogenic assays were carried out to calculate the SF2 for each cell line (shown in the graph). Equal cell numbers were loaded onto an SDA-PAGE gel and Brk levels detected by western blotting (lower panels). Anti-GAPDH was used as a loading control.

expression and the activity of the key cell cycle regulator ATM, which is central to the repair of DNA DSB.

The Brk kinase is an attractive target for study within the context of breast cancer treatment given that up to $85 \%$ of all invasive ductal breast cancers over-express Brk [23]. While Brk has been demonstrated to contribute to tumor progression via a number of mechanisms such as enhanced proliferation [18], elevated cancer cell migration and protection from autophagy [9], the role of Brk in mediating the response to cytotoxic radiotherapy has not been investigated. However, this is a pertinent enquiry vis-à-vis the potential modulation of ATM activity via Brk. Brk over-expression has previously been linked to enhanced ErbB signalling [8,23,14], a pathway with known links to radiation sensitivity [23]. Moreover it has been recently demonstrated that enhanced mTOR signalling in the context of childhood sarcoma mediated down-regulation of ATM by microRNA [22]. Thus it may be speculated that Brk over-expression may lead to breast cancer cell line hypersensitivity to radiation due to the lack of the G1 cell cycle checkpoint brought about by ATM deficiency. We examined the response of two basal-type cell lines, MDA-MB-157 and MDA-MB-468 to increasing doses of radiation in a clonogenic assay. Each cell line had been transfected with a plasmid containing either a cDNA encoding either wild type Brk (Brk-WT), Brk with a mutation in the tyrosine kinase domain of the gene (Brk-KM) or the empty vector. In the MDAMB-157 Brk-WT cell line there was enhanced resistance to increasing doses of gamma radiation when compared to the Brk-KM and vector cell lines. By comparing the SF2 values (a sub-lethal dose) the wild-type Brk-expressing cell line was significantly more resistant than the other two counterpart cell lines (Student's unpaired t-test $\mathrm{p}>0.05$ ).

In the MDA-MB-468 cell lines the Brk-WT and Brk-KM cell lines exhibited similar radiation sensitivity and a comparison of the SF2 values for these cells lines revealed no significant difference in sensitivity (Student's unpaired t-test $\mathrm{p}=0.385$ ). The difference in sensitivity between MDA-MB-468 and MDA-MB-157 in response to radiation in the presence of wild-type Brk could be explained, in part, by the signalling pathways activated in each parental cell lines. Despite both cell lines being characterised as triple negative [24], MDAMB-468 are known to have amplified levels of EGFR plus mutations in the PI3-K/AKT inhibitor PTEN [25]. Therefore addition of Brk may have little impact on a cell line with already elevated EGFR signalling. On the other hand the MDA-MB-157 cell line is not reported to have any abnormalities in EGFR signalling, suggesting that Brk expression would be more likely to impact on EGFR signalling in these cells. Given that EGFR signalling is linked to radiation responses, increased Brk might lead to increased survival of these cells in a kinase dependent manner.

In addition to analysing the radiation sensitivity in a clonogenic assay, we also sought to evaluate DNA DSB repair in the two cell line groups. Previous observations by our group and others reveal that hypersensitivity to ionising radiation is often associated with a defect or delayed repair of DSB. This is exemplified in cells derived from individuals with inherited mutations in genes controlling the recognition and repair of DNA double strand break repair. For example in A-T cells there is a deficiency in the repair of ionising radiation induced DSB shown by a persistence of $\gamma-\mathrm{H} 2 \mathrm{~A} \Xi$ foci in the nuclei of cells when compared to normal cells [21]. Based upon these observations, we might expect that the breast cancer cells lines in this study which are sensitive to radiation such as the MDA-MB-157 Brk$\mathrm{KM}$ and vector cell lines (when compared to the Brk-WT derivative), may exhibit a defect in the repair of DSB in the foci assay. We do observe induction of foci in all cell lines after radiation exposure, there are no significant differences in the kinetics of repair over a 24 hour period $(\mathrm{p}<0.05)$ and a similar pattern is observed with the MDA-MB-468 cells.

To determine if there was a potential link between Brk activity and ATM phosphorylation status in the cell lines we investigated the levels of total and phosphorylated forms of ATM in our six cell line derivatives. The principal phosphorylation site of ATM is the phosphoserine794 which is activated by proteins such as CDK5 and the auto-phosphorylation site serine1891 [26]. Our analysis of total ATM and phosphoserine794 ATM again revealed minor differences in the levels of both protein forms before and after exposure to $2 \mathrm{~Gy}$ 
Citation: Bourton EC, Hussain H, Plowman PN, Harvey AJ, Parris CN (2015) Radiosensitivity of Human Breast Cancer Cell Lines Expressing the Breast Tumor Kinase (Brk). J Cancer Sci Ther 7: 095-101. doi:10.4172/1948-5956.1000331

gamma radiations. Therefore this data suggests that there may be no direct molecular or biochemical link between Brk and ATM signalling.

Finally, having looked at Brk expression in relation to clonogenic survival following exposure to 2 Gy radiation, we found no direct correlation between Brk levels and cell survival. Combined with the data above, we hypothesise that the effect of Brk on radiation responses appears to be mediated by signalling outputs other than DSB repair.

In summary, we have conducted an investigation into the radiosensitivity of breast cancer cells lines over-expressing the Brk tumor kinase. We find no consistent alteration in cellular radiosensitivity in cells expressing either wild type or kinase-mutated Brk. Finally we do conceded that our study focussed on two sets of breasty cancer cell lines which have been genetically modified to over-express wild type or mutant Brk and a more comprehensive study of Brk expression and radiation sensitivity in more cell lines is required. Also a clinical study examining the response of breast cancers to radiotherapy visà-vis Brk expression would establish fully the relationship between Brk expression and radiotherapy.

\section{Acknowledgements}

We acknowledge the help of Miss Connie Sarah Parris and Miss Jennifer Anne Parris for assistance with clonogenic assays.

\section{Declaration of interest}

The authors report no conflicts of interest.

This work was supported by a grant from The Bart's Charity (Grant Number: 419/2071), 12 Cock Lane, London EC1A 9BU

\section{References}

1. CRUK Statistics (2014).

2. Tryfonidis K, Senkus E, Cardoso MJ, Cardoso F (2015) Management of locally advanced breast cancer-perspectives and future directions. Nat Rev Clin Oncol 12: $147-162$.

3. The Best Treatment (2013) Your Guide to breast cancer treatment in England and Wales. Breakthrough Breast Cancer Edition 3.

4. The Best Treatment (2012) Your Guide to breast cancer treatment in Scotland. Breakthrough Breast Cancer Edition 2.

5. Genovesi D, Mazzilli L, Trignani M, DI Tommaso M, Nuzzo A, et al. (2014) Developing a decision-making model based on an interdisciplinary oncological care group for the management of colorectal cancer. Anticancer Res 34: 25252531.

6. Harvey A, Burmi R (2011) Future Therapeutic Strategies: Implications for Brk Targeting. In: Gunduz E and Gundez M eds. Breast Cancer - Current and Alternative Therapeutic Modalities. InTech 413-434.

7. Aubele M, Auer G, Walch AK, Munro A, Atkinson MJ, et al. (2007) PTK (protein tyrosine kinase)- 6 and HER2 and 4, but not HER1 and 3 predict long-term survival in breast carcinomas. $\mathrm{Br} \mathrm{J}$ Cancer 96: 801-807.

8. Ostrander JH, Daniel AR, Lofgren K, Kleer CG, Lange CA (2007) Breast tumor kinase (protein tyrosine kinase 6 ) regulates heregulin-induced activation of ERK5 and p38 MAP kinases in breast cancer cells. Cancer Res 67: 4199-4209.

9. Harvey AJ, Pennington CJ, Porter S, Burmi RS, Edwards DR, et al. (2009)
Brk protects breast cancer cells from autophagic cell death induced by loss of anchorage. Am J Pathol 175: 1226-1234.

10. Haegebarth A, Perekatt AO, Bie W, Gierut JJ, Tyner AL (2009) Induction of protein tyrosine kinase 6 in mouse intestinal crypt epithelial cells promotes DNA damage-induced apoptosis. Gastroenterology 137: 945-954.

11. Gierut JJ, Mathur PS, Bie W, Han J, Tyner AL (2012) Targeting protein tyrosine kinase 6 enhances apoptosis of colon cancer cells following DNA damage. Mol Cancer Ther 11: 2311-2320.

12. Kamalati T, Jolin HE, Mitchell PJ, Barker KT, Jackson LE, et al. (1996) Brk a breast tumor-derived non-receptor protein-tyrosine kinase, sensitizes mammary epithelial cells to epidermal growth factor. J Biol Chem 271: 30956 30963.

13. Lauring J, Park BH, Wolff AC (2013) The phosphoinositide-3-kinase-Akt-mTOR pathway as a therapeutic target in breast cancer. J Natl Compr Canc Netw 11: 670-678.

14. Shen C, Houghton PJ (2013) The mTOR pathway negatively controls ATM by up-regulating miRNAs. Proc Natl Acad Sci U S A 110: 11869-11874.

15. Lavin MF, Shiloh Y (1997) The genetic defect in ataxia-telangiectasia. Annu Rev Immunol 15: 177-202.

16. Neve RM, Chin K, Fridlyand J, Yeh J, Baehner FL, et al. (2006) A collection of breast cancer cell lines for the study of functionally distinct cancer subtypes. Cancer Cell 10: 515-527.

17. Barker KT, Jackson LE, Crompton MR (1997) BRK tyrosine kinase expression in a high proportion of human breast carcinomas. Oncogene 15: 799-805.

18. Harvey AJ, Crompton MR (2003) Use of RNA interference to validate Brk as a novel therapeutic target in breast cancer: Brk promotes breast carcinoma cell proliferation. Oncogene 22: 5006-5010.

19. Ulus-Senguloglu G, Arlett CF, Plowman PN, Parnell J, Patel N, et al. (2012) Elevated expression of artemis in human fibroblast cells is associated with cellular radiosensitivity and increased apoptosis. Br J Cancer 107: 1506-1513.

20. Bourton EC, Plowman PN, Zahir SA, Senguloglu GU, Serrai H, et al. (2012) Multispectral imaging flow cytometry reveals distinct frequencies of $\hat{i}^{3}-\mathrm{H} 2 \mathrm{AX}$ foci induction in DNA double strand break repair defective human cell lines. Cytometry A 81: 130-137.

21. Bourton EC, Plowman PN, Harvey AJ, Adam Zahir S, Parris CN (2013) The PARP-1 inhibitor Olaparib causes retention of $\mathrm{y}-\mathrm{H} 2 \mathrm{~A} \equiv$ foci in BRCA1 heterozygote cells following exposure to gamma radiation. $J$ Cancer Ther 4 44-52.

22. Kamalati T, Jolin HE, Fry MJ, Crompton MR (2000) Expression of the BRK tyrosine kinase in mammary epithelial cells enhances the coupling of EGF signalling to PI 3-kinase and Akt, via erbB3 phosphorylation. Oncogene 19 5471-5476.

23. Liang K, Lu Y, Jin W, Ang KK, Milas L, et al. (2003) Sensitization of breast cancer cells to radiation by trastuzumab. Mol Cancer Ther 2: 1113-1120.

24. Lehmann BD, Bauer JA, Chen X, Sanders ME, Chakravarthy AB, et al. (2011) Identification of human triple-negative breast cancer subtypes and preclinical models for selection of targeted therapies. J Clin Invest 121: 2750-2767.

25. Bamford S, Dawson E, Forbes S, Clements J, Pettett R, et al. (2004) The COSMIC (Catalogue of Somatic Mutations in Cancer) database and website. Br J Cancer 91: 355-358.

26. Lee JH, Paull TT (2007) Activation and regulation of ATM kinase activity in response to DNA double-strand breaks. Oncogene 26: 7741-7748. 